\title{
MULTIPLICITY OF THE LOWEST EIGENVALUE OF NON-COMMUTATIVE HARMONIC OSCILLATORS
}

\author{
Fumio HIROSHIMA and Itaru SASAKI
}

(Received 18 July 2012 and revised 9 October 2012)

\begin{abstract}
The multiplicity of the lowest eigenvalue $E$ of the so-called non-commutative harmonic oscillator $Q(\alpha, \beta)$ is studied. It is shown that $E$ is simple for $\alpha$ and $\beta$ in some region.
\end{abstract}

\section{Definition and main results}

Recently special attention has been paid to studying the spectrum of self-adjoint operators with non-commutative coefficients. This is considered in not only mathematics but also physics experiments. A historically important model is the Dirac operator, and the Rabi model and the Jaynes-Cumming model are prevalent in cavity quantum electrodynamics (see [HH12] and references therein). The non-commutative harmonic oscillator is a quantum system defined by the Hamiltonian:

$$
Q=Q(\alpha, \beta)=A \otimes\left(-\frac{1}{2} \frac{d^{2}}{d x^{2}}+\frac{1}{2} x^{2}\right)+J \otimes\left(x \frac{d}{d x}+\frac{1}{2}\right),
$$

where $A=\left(\begin{array}{cc}\alpha & 0 \\ 0 & \beta\end{array}\right), J=\left(\begin{array}{cc}0 & -1 \\ 1 & 0\end{array}\right)$, and $\alpha, \beta>0$ parameters with $\alpha \beta>1$. Operator $Q$ defines a positive self-adjoint operator acting in the Hilbert space $\mathcal{H}=\mathbb{C}^{2} \otimes L^{2}(\mathbb{R})$.

The non-commutative harmonic oscillator $Q$ has been introduced by Parmeggiani and Wakayama [PW01, PW02a, PW02b, PW03], and the spectral property of $Q$ is considered in [Par04, Par06, Par08a] from the pseudo-differential calculus point of view. It can be seen that $Q$ has purely discrete spectrum $\lambda_{1} \leq \lambda_{2} \leq \cdots \leq \lambda_{n} \leq \cdots \uparrow \infty$, where the eigenvalues are counted with multiplicity. One can define the so-called spectral zeta function associated with $Q$ as

$$
\zeta_{Q}(s)=\sum_{n=1}^{\infty} \frac{1}{\lambda_{n}^{s}}
$$

When $\alpha=\beta, Q$ is unitarily equivalent to the direct sum of harmonic oscillators, and $\lambda_{2 m-1}=\lambda_{2 m}=\sqrt{\alpha^{2}-1}\left(m+\frac{1}{2}\right)$, and thus $\zeta_{Q}$ with $\alpha \neq \beta$ can be regarded as a $q$-deform of the Riemann zeta-function. Analytic properties of the spectral zeta-function are studied in [IW05a, IW05b, IW07, KW06, KW07, KY09]. Furthermore, it is also known that the set of odd eigenvectors of non-commutative harmonic oscillator is deeply related to the set of some

2010 Mathematics Subject Classification: Primary 35P15; Secondary 35P05.

Keywords: lowest eigenvalue; multiplicity; non-commutative harmonic oscillator.

(C) 2013 Faculty of Mathematics, Kyushu University 
solutions of the Heun differential equation [IW05b, Och01, Och04]:

$$
\frac{\partial^{2}}{\partial w^{2}} f+\left(\frac{1-n}{w}+\frac{-n}{w-1}+\frac{n+3 / 2}{w-a}\right) \frac{\partial}{\partial w} f+\frac{-(3 / 2) n w-q}{w(w-1)(w-a)} f=0,
$$

where $n \in \mathbb{N} \cup\{0\}, a \in \mathbb{C}$ with $|a|<1$ and $q \in \mathbb{C}$.

In this paper we concentrate on the study of the lowest eigenvalue $\lambda_{1}$ of $Q$. We set

$$
E=\lambda_{1}
$$

and $p=-i d / d x$. In particular, we are interested in determining the dimension of $\operatorname{Ker}(Q-E)$. The eigenvector associated with the lowest eigenvalue is called the ground state. In the case of $\alpha=\beta$, as is mentioned above, $Q$ can be diagonalized as $Q \cong\left(\begin{array}{ll}h & 0 \\ 0 & h\end{array}\right)$ with

$$
h=\frac{1}{2} p^{2}+\frac{\alpha^{2}-1}{2} x^{2},
$$

where $\cong$ denotes the unitary equivalence. Then all of the eigenvalues of $Q(\alpha, \alpha)$ are twofold degenerate. In particular, its lowest eigenvalue

$$
E_{0}=\frac{1}{2} \sqrt{\alpha^{2}-1}
$$

is two-fold degenerate. In the general case, $\alpha \neq \beta$, the so-called Ichinose-Wakayama (IW) bound is established in [IW07] (see also [Par10]):

$$
\left(j-\frac{1}{2}\right) \min \{\alpha, \beta\} \sqrt{\frac{\alpha \beta-1}{\alpha \beta}} \leq \lambda_{2 j-1} \leq \lambda_{2 j} \leq\left(j-\frac{1}{2}\right) \max \{\alpha, \beta\} \sqrt{\frac{\alpha \beta-1}{\alpha \beta}}
$$

for $j \in \mathbb{N}$. By this inequality we see that the multiplicity of $E$ is at most two if $\beta<3 \alpha$ or $\alpha<3 \beta$.

Furthermore, beyond the above results one may expect that $E$ is simple for $\alpha \neq \beta$. In [NNW02] it is numerically shown that $E$ is simple for $\alpha \neq \beta$ and, in [Par04], the simplicity is proven but only for sufficiently large $\alpha \beta$. It is then mentioned in [Par08b, 8.3 Notes] that the determination of the multiplicity of the lowest eigenvalue should be explored.

In this paper we show that:

(a) $\quad E$ is at most twofold degenerate for $(\alpha, \beta) \in(2, \infty) \times(2, \infty)$;

(b) $E$ is simple for some region of $\alpha$ and $\beta$.

In order to prove (a), we apply the method in [Hir05], where the so-called pull-through formula [GJ68] is a key ingredient. The second result (b) consists of two estimates. The first is for large $|\beta-\alpha|$ and the second for small $|\beta-\alpha|$ but $\alpha \neq \beta$. The first case is proven in a similar manner to (a) and the second by the regular perturbation theory of discrete spectrum.

Let $\mathscr{G}=\operatorname{Ker}(Q-E)$ be the set of ground states. Let $L_{+} \subset L^{2}(\mathbb{R})$ (respectively $L_{-}$) be the set of even functions (respectively odd functions). We define $\mathcal{H}_{ \pm}=\mathbb{C}^{2} \otimes L_{ \pm}$. Since $Q$ conserves the parity, $Q$ is reduced by $\mathcal{H}_{ \pm}$. Set $Q\left\lceil\mathcal{H}_{ \pm}=Q_{ \pm}\right.$and then $Q=Q_{+} \oplus Q_{-}$.

Theorem 1.1. Suppose that $(\alpha, \beta) \in(2, \infty) \times(2, \infty)$. Then $\operatorname{dim} \mathscr{G} \leq 2$ and $\mathscr{G} \subset \mathcal{H}_{+}$. That is, the multiplicity of $E$ is at most two and ground states are even functions.

We can furthermore show that $E$ is simple. 
TheOREM 1.2. Suppose that $\beta>\alpha>2$ and

$$
\frac{1}{2}>\left(\frac{1}{2} \beta-E\right)^{-2} \frac{1}{\alpha^{2}-1}+\frac{1}{\alpha^{2}-1} .
$$

Then E is simple.

Condition (1.6) includes the implicit value $E$. Let

$$
E_{\text {upper }}=\frac{\sqrt{\alpha \beta} \sqrt{\alpha \beta-1}}{\alpha+\beta+|\alpha-\beta|\left((\alpha \beta-1)^{1 / 4} / \sqrt{\alpha \beta}\right) \operatorname{Re} \rho},
$$

where $\rho^{2}=\sqrt{\alpha \beta-1}-i$ with $\operatorname{Re} \rho>0$, i.e. $\operatorname{Re} \rho=\sqrt{\sqrt{\alpha \beta}(\sqrt{\alpha \beta-1}+1) / 2}$. The bound $E<E_{\text {upper }}$ holds. See [Par08b, Theorem 8.2.1] and [Par10]. This upper bound is better than the IW bound (1.5). Combining this with (1.6) we have the following corollary.

COROLlary 1.3. Suppose that $\beta>\alpha>2$ and

$$
\frac{1}{2}>\left(\frac{1}{2} \beta-E_{\text {upper }}\right)^{-2} \frac{1}{\alpha^{2}-1}+\frac{1}{\alpha^{2}-1} .
$$

Then E is simple.

Theorem 1.2 does not hold for $(\alpha, \beta)$ in a neighborhood of the diagonal line on $\alpha-\beta$ plane. See Figure 1. However, we can show that $E$ is simple for $\alpha$ and $\beta$ in a suitable neighborhood of the diagonal line. We define $g_{1}, \ldots, g_{4}$ by

$$
\begin{aligned}
& g_{1}=(\alpha-1)^{-1}\left(3+\frac{\sqrt{3}}{\sqrt{\alpha^{2}-1}}\right), \\
& g_{2}=\frac{\sqrt{\alpha^{2}-1}}{2\left|\sqrt{\alpha^{2}-1}-\lambda_{2}\right|} g_{1}^{2}, \\
& g_{3}=\frac{\alpha}{2 \sqrt{\alpha^{2}-1}}, \\
& g_{4}=\frac{\left(\sqrt{\alpha^{2}-1}\right)^{3 / 2}}{4 \alpha^{3 / 2}} .
\end{aligned}
$$

THEOREM 1.4. Let $\varepsilon=\beta-\alpha$. Assume that $\beta>\alpha>1, \sqrt{\beta^{2}-1} \leq 3 \sqrt{\alpha^{2}-1}$ and $\varepsilon^{2} g_{2}<1 / 2$. Let

$$
\begin{aligned}
& \kappa(\varepsilon)=E_{0} g_{1}^{2}+\varepsilon g_{2}\left(E_{0} g_{1}+g_{3}+g_{4}\right)+\varepsilon^{2} 2 E_{0} g_{1}^{2} g_{2}+\varepsilon^{3} 2 E_{0} g_{1} g_{2}^{2}, \\
& \ell(\varepsilon)=\left(1-\varepsilon^{2} g_{2}\right) \sqrt{1-2 \varepsilon^{2} g_{2}^{2}} .
\end{aligned}
$$

Then

$$
\left|\lambda_{1}-\lambda_{2}\right| \geq \frac{2 \varepsilon}{\ell(\varepsilon)}\left(g_{4}-\varepsilon \kappa(\varepsilon)\right) .
$$

In particular when $\varepsilon \kappa(\varepsilon)<g_{4}, E$ is simple.

Note that we know the bound $\lambda_{2} \leq(\beta / 2)(\sqrt{\alpha \beta-1} / \sqrt{\alpha \beta})$ by the IW bound. Then, the region of $\alpha, \beta$ satisfying $\varepsilon \kappa(\varepsilon)<g_{4}$, includes a wedge-shaped region illustrated in Figure 2 , where we also drew the case of $\alpha>\beta$. 


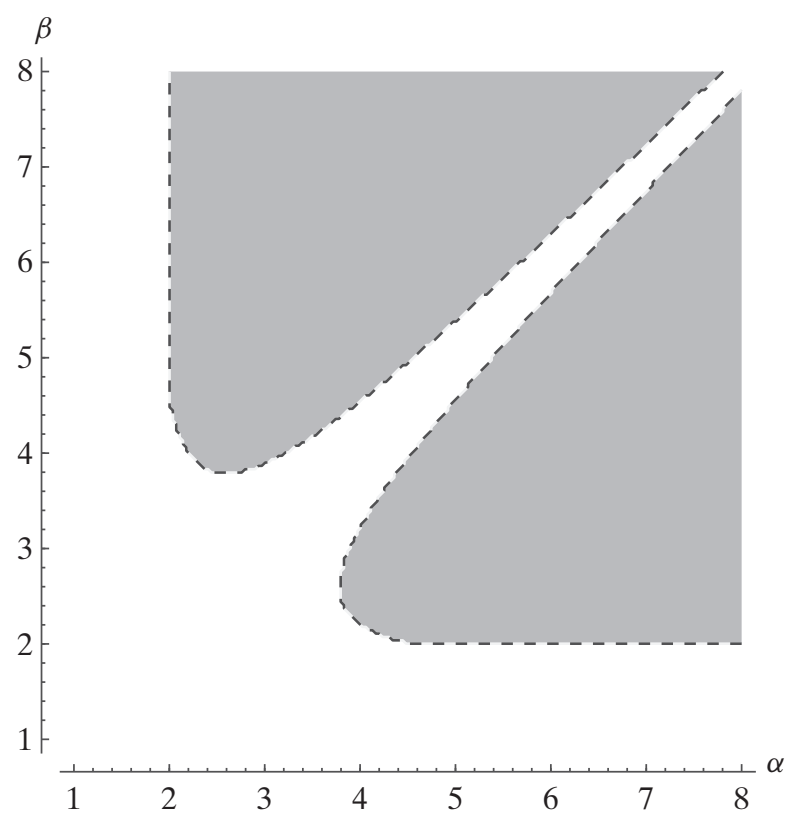

FIGURE 1. The region satisfying (1.7). (The case of $\alpha>\beta$ is also drawn.)

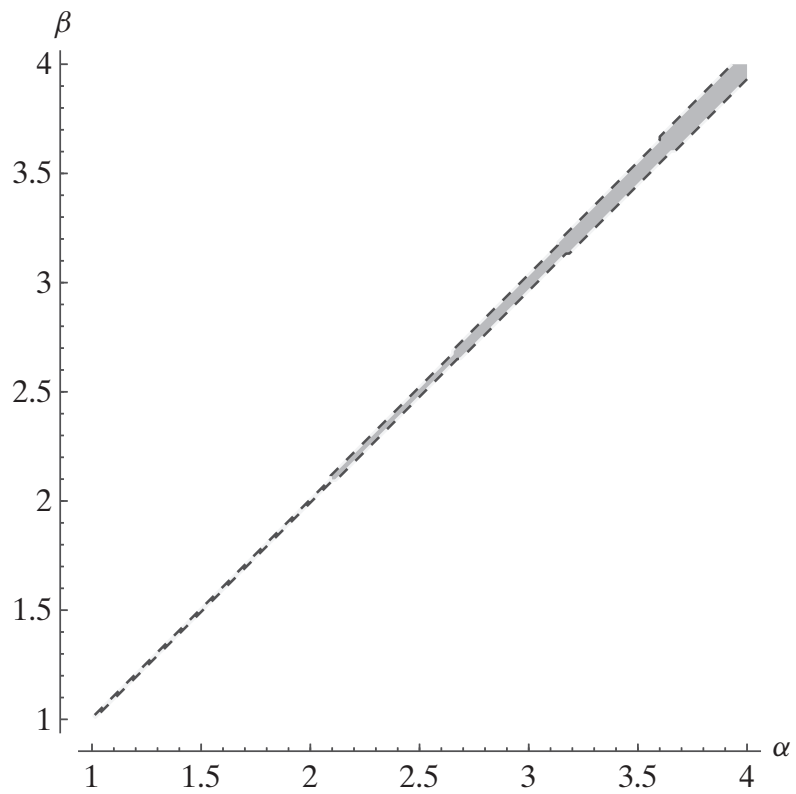

FIGURE 2. A wedge-shaped region included in the region satisfying $\varepsilon \kappa(\varepsilon)<g_{4}$. 


\section{Proofs of theorems}

\subsection{Proof of Theorem 1.1}

In the following we omit the symbol $\otimes$ for the notational simplicity, and we can suppose that $\alpha<\beta$ without loss of generality. Let

$$
N=\frac{1}{2}\left(\begin{array}{ll}
1 & 0 \\
0 & 1
\end{array}\right)\left(p^{2}+x^{2}-1\right) .
$$

The spectrum of $N$ is $\sigma(N)=\{0\} \cup \mathbb{N}$ and the multiplicity of each eigenvalue is two. Let $a=\frac{1}{\sqrt{2}}(x+i p)$ and $a^{*}=\frac{1}{\sqrt{2}}(x-i p)$. They satisfy canonical commutation relations $\left[a, a^{*}\right]=1$ and $[a, a]=\left[a^{*}, a^{*}\right]=0$, and we have $a^{*} a=N$.

Proof of Theorem 1.1. In terms of $a$ and $a^{*}$, the operator $Q$ can be realized as

$$
Q=a^{*} A a+\frac{1}{2} A+\frac{1}{2}\left(a J a-a^{*} J a^{*}\right) .
$$

Let $\varphi_{\mathrm{g}} \in \mathscr{G}$. We have $(Q-E) a \varphi_{\mathrm{g}}=[Q, a] \varphi_{\mathrm{g}}$. Since $[Q, a]=-A a+J a^{*}$ by canonical commutation relation, we have $(Q-E) a \varphi_{\mathrm{g}}=\left(-A a+J a^{*}\right) \varphi_{\mathrm{g}}$ and then $(Q-E+A) a \varphi_{\mathrm{g}}$ $=J a^{*} \varphi_{\mathrm{g}}$. Note that $Q-E+A \geq \alpha>0$. We have

$$
a \varphi_{\mathrm{g}}=(Q-E+A)^{-1} J a^{*} \varphi_{\mathrm{g}} .
$$

Taking the norm on both sides above, we have

$$
\left\|a \varphi_{\mathrm{g}}\right\|^{2} \leq \frac{1}{\alpha^{2}}\left\|a^{*} \varphi_{\mathrm{g}}\right\|^{2}
$$

Since $\left\|a \varphi_{\mathrm{g}}\right\|^{2}=\left\langle\varphi_{\mathrm{g}}, N \varphi_{\mathrm{g}}\right\rangle$ and $\left\|a^{*} \varphi_{\mathrm{g}}\right\|^{2}=\left\langle\varphi_{\mathrm{g}}, N \varphi_{\mathrm{g}}\right\rangle+\left\|\varphi_{\mathrm{g}}\right\|^{2}$, we see that

$$
\left\langle\varphi_{\mathrm{g}}, N \varphi_{\mathrm{g}}\right\rangle \leq \frac{1}{\alpha^{2}-1}\left\|\varphi_{\mathrm{g}}\right\|^{2}
$$

Let $\mathrm{P}_{\Omega}$ be the projection onto $\operatorname{ker} N=\operatorname{ker} a$. Note that $N+\mathrm{P}_{\Omega} \geq 1$. Let $\mathscr{G}=\mathscr{G}_{+} \oplus \mathscr{G}_{-}$, where $\mathscr{G}_{ \pm}=\mathscr{G} \cap \mathcal{H}_{ \pm}$. Let $\mathrm{P}^{ \pm}$be the projection onto $\mathscr{G}_{ \pm}$. Then, by (2.4), we have

$$
\mathrm{P}^{+} \mathrm{P}_{\Omega} \mathrm{P}^{+} \geq \mathrm{P}^{+}(1-N) \mathrm{P}^{+} \geq\left(1-\frac{1}{\alpha^{2}-1}\right) \mathrm{P}^{+} .
$$

Taking the trace of both sides, we have

$$
2 \geq \operatorname{Tr}\left(\mathrm{P}^{+} \mathrm{P}_{\Omega} \mathrm{P}^{+}\right) \geq \frac{\alpha^{2}-2}{\alpha^{2}-1} \operatorname{Tr} \mathrm{P}^{+} .
$$

Thus, we have the bound

$$
\operatorname{dim} \operatorname{ker} \mathrm{P}^{+} \leq 2 \frac{\alpha^{2}-1}{\alpha^{2}-2}
$$

Then the right-hand side above is less than three for $\alpha>2$. Then $\operatorname{dim} \mathscr{G}^{+} \leq 2$. Similarly but replacing $\mathrm{P}^{+}$with $\mathrm{P}^{-}$, we can also see that

$$
\mathrm{P}^{-} \mathrm{P}_{\Omega} \mathrm{P}^{-} \geq \mathrm{P}^{-}(1-N) \mathrm{P}^{-} \geq\left(1-\frac{1}{\alpha^{2}-1}\right) \mathrm{P}^{-} .
$$

Note that $\mathrm{P}^{-} \mathrm{P}_{\Omega} \mathrm{P}^{-}=0$, since $\mathrm{P}_{\Omega}$ is the projection to the set of even functions. Then we have

$$
0=\operatorname{Tr}\left(\mathrm{P}^{-} \mathrm{P}_{\Omega} \mathrm{P}^{-}\right) \geq \frac{\alpha^{2}-2}{\alpha^{2}-1} \operatorname{Tr} \mathrm{P}^{-} .
$$

In particular, for $\alpha>\sqrt{2}$, the dimension of $\mathscr{G}_{-}$equals zero. Then the theorem follows. 


\subsection{Proof of Theorem 1.2}

In this section we show that the lowest eigenvalue is simple. The strategy is parallel to that of the previous section but with $\mathrm{P}^{+}$replaced by a projector $\mathrm{R}$ with dimension $\mathrm{Ran} \mathrm{R}=1$. Let $\sigma_{1}, \sigma_{2}, \sigma_{3}$ be the $2 \times 2$ Pauli matrices given by

$$
\sigma_{1}=\left(\begin{array}{cc}
0 & 1 \\
1 & 0
\end{array}\right), \quad \sigma_{2}=\left(\begin{array}{cc}
0 & -i \\
i & 0
\end{array}\right), \quad \sigma_{3}=\left(\begin{array}{cc}
1 & 0 \\
0 & -1
\end{array}\right)
$$

Proof of Theorem 1.2. The Hamiltonian $Q$ can be written in the form

$$
Q=\frac{1}{2} A\left(p^{2}+x^{2}\right)+\frac{1}{2} \sigma_{2}(p x+x p) .
$$

We set $M=\frac{1}{2}\left(1+\sigma_{3}\right)=\left(\begin{array}{ll}1 & 0 \\ 0 & 0\end{array}\right), M^{\perp}=\frac{1}{2}\left(1-\sigma_{3}\right)=\left(\begin{array}{ll}0 & 0 \\ 0 & 1\end{array}\right)$ and $\mathrm{R}=M^{\perp} \mathrm{P}_{\Omega}$. Then we have

$$
(Q-E) \mathrm{R} \varphi_{\mathrm{g}}=M^{\perp}\left[Q, \mathrm{P}_{\Omega}\right] \varphi_{\mathrm{g}}+\left[Q, M^{\perp}\right] \mathrm{P}_{\Omega} \varphi_{\mathrm{g}} .
$$

The commutator $\left[Q, M^{\perp}\right]$ can be computed as

$$
\left[Q, M^{\perp}\right]=-\frac{1}{2}\left(a a-a^{*} a^{*}\right) \sigma_{1} .
$$

Thus, we have

$$
[Q, \mathrm{R}]=M^{\perp} \frac{i}{2} \sigma_{2}\left[a a-a^{*} a^{*}, \mathrm{P}_{\Omega}\right]+\frac{1}{2} a^{*} a^{*} \sigma_{1} \mathrm{P}_{\Omega},
$$

where we used the fact that $a \mathrm{P}_{\Omega}=0$. Hence, we have

$$
\begin{aligned}
\left\langle\mathrm{R} \varphi_{\mathrm{g}},(Q-E) \mathrm{R} \varphi_{\mathrm{g}}\right\rangle & =\left\langle\varphi_{\mathrm{g}}, \frac{i}{2} \mathrm{R} M^{\perp} \sigma_{2}\left[a a-a^{*} a^{*}, \mathrm{P}_{\Omega}\right] \varphi_{\mathrm{g}}\right\rangle+\frac{1}{2}\left\langle\varphi_{\mathrm{g}}, \mathrm{R} a^{*} a^{*} \sigma_{1} \mathrm{P}_{\Omega} \varphi_{\mathrm{g}}\right\rangle \\
& =\frac{i}{2}\left\langle\varphi_{\mathrm{g}}, \mathrm{R} \sigma_{2} a^{2} \varphi_{\mathrm{g}}\right\rangle .
\end{aligned}
$$

On the other hand, $\mathrm{R}(Q-E) \mathrm{R}=\left(\frac{1}{2} \beta-E\right) \mathrm{R}^{2}$. Then (2.4) and $\left\|a^{*} \mathrm{R} \varphi_{\mathrm{g}}\right\|=\left\|\mathrm{R} \varphi_{\mathrm{g}}\right\|$ yield that

$$
\left(\frac{1}{2} \beta-E\right)\left\|\mathrm{R} \varphi_{\mathrm{g}}\right\|^{2} \leq \frac{1}{2}\left\|a^{*} \mathrm{R} \varphi_{\mathrm{g}}\right\|\left\|\sigma_{2} a \varphi_{\mathrm{g}}\right\| \leq \frac{1}{\sqrt{\alpha^{2}-1}}\left\|\mathrm{R} \varphi_{\mathrm{g}}\right\|\left\|\varphi_{\mathrm{g}}\right\| .
$$

Therefore,

$$
\left\|\mathrm{R} \varphi_{\mathrm{g}}\right\|^{2} \leq\left(\frac{1}{2} \beta-E\right)^{-2} \frac{1}{\alpha^{2}-1}\left\|\varphi_{\mathrm{g}}\right\|^{2} .
$$

Since $M+M^{\perp}=1$, it holds that $\mathrm{P}_{\Omega} M+\mathrm{R}+N \geq 1$. Then, by using (2.4) we have

$$
\mathrm{P}\left(\mathrm{P}_{\Omega} M\right) \mathrm{P} \geq \mathrm{P}\left(1-\mathrm{P}_{\Omega} M^{\perp}-N\right) \mathrm{P} \geq\left(1-\left(\frac{1}{2} \beta-E\right)^{-2} \frac{1}{\alpha^{2}-1}-\frac{1}{\alpha^{2}-1}\right) \mathrm{P},
$$

where $P=P^{+}+P^{-}$is the orthogonal projection onto $\mathscr{G}$. Taking the trace of both sides above, we have

$$
1 \geq\left(1-\left(\frac{1}{2} \beta-E\right)^{-2} \frac{1}{\alpha^{2}-1}-\frac{1}{\alpha^{2}-1}\right) \operatorname{Tr} \mathrm{P},
$$

and the theorem follows. 


\subsection{Proof of Theorem 1.4}

Recall that $\varepsilon=\beta-\alpha$. In this section, we fix an arbitrary $\alpha>1$ and set

$$
Q(\alpha, \beta)=Q=Q(\varepsilon)=Q_{0}+\varepsilon V,
$$

where $Q_{0}=Q(\alpha, \alpha)$ and $V=\frac{1}{2}\left(\begin{array}{ll}0 & 0 \\ 0 & 1\end{array}\right)\left(p^{2}+x^{2}\right)$.

LEMMA 2.1. For all $\Phi \in D\left(Q_{0}\right)$, it follows that

$$
\|V \Phi\| \leq(\alpha-1)^{-1}\left\|Q_{0} \Phi\right\|+\frac{\sqrt{3}}{2}(\alpha-1)^{-1}\|\Phi\| .
$$

Proof. One can show that $\|(p x+x p) u\|^{2} \leq\left\|\left(p^{2}+x^{2}\right) u\right\|^{2}+3\|u\|^{2}$. Since $\sigma_{2}^{2}=1$, we have

$$
\begin{aligned}
\left\|Q_{0} \Phi\right\| & \geq \alpha\left\|\frac{1}{2}\left(p^{2}+x^{2}\right) \Phi\right\|-\left\|\frac{1}{2}(p x+x p) \Phi\right\| \\
& \geq \alpha\left\|\frac{1}{2}\left(p^{2}+x^{2}\right) \Phi\right\|-\frac{1}{2}\left(\left\|\left(p^{2}+x^{2}\right) \Phi\right\|^{2}+3\|\Phi\|^{2}\right)^{1 / 2},
\end{aligned}
$$

and, hence,

$$
\left\|Q_{0} \Phi\right\| \geq \frac{\alpha-1}{2}\left\|\left(p^{2}+x^{2}\right) \Phi\right\|-\frac{\sqrt{3}}{2}\|\Phi\| .
$$

Noting that $\|V \Phi\| \leq \frac{1}{2}\left\|\left(p^{2}+x^{2}\right) \Phi\right\|$ we have the bound (2.17).

By (1.5) or the sandwich estimate $Q(\alpha, \alpha) \leq Q(\alpha, \beta) \leq Q(\beta, \beta)$ we have the bounds

$$
\frac{\sqrt{\alpha^{2}-1}}{2} \leq \lambda_{1} \leq \lambda_{2} \leq \frac{\sqrt{\beta^{2}-1}}{2} \text { and } \frac{3}{2} \sqrt{\alpha^{2}-1} \leq \lambda_{3}
$$

(with repetition according to multiplicity). When $\sqrt{\beta^{2}-1} \leq 3 \sqrt{\alpha^{2}-1}, Q$ has exactly two eigenvalues in the interval $\left[\frac{1}{2} \sqrt{\alpha^{2}-1}, \frac{1}{2} \sqrt{\beta^{2}-1}\right]$. Let $C$ be the closed disk centered at $\sqrt{\alpha^{2}-1} / 2$ with the radius $\sqrt{\alpha^{2}-1} / 2$ in the complex plane:

$$
C=\left\{\frac{\sqrt{\alpha^{2}-1}}{2}+r e^{i \theta} \in \mathbb{C} \mid 0 \leq r \leq \frac{\sqrt{\alpha^{2}-1}}{2}, 0 \leq \theta \leq 2 \pi\right\} .
$$

Thus, $Q$ has exactly two eigenvalues, $\lambda_{1}$ and $\lambda_{2}$, inside of $C$.

LEMMA 2.2. We have $\left\|V\left(Q_{0}-z\right)^{-1}\right\| \leq g_{1}$ for all $z \in \partial C$.

Proof. By Lemma 2.1, we have

$$
\left\|V\left(Q_{0}-z\right)^{-1}\right\| \leq(\alpha-1)^{-1}\left\|Q_{0}\left(Q_{0}-z\right)^{-1}\right\|+\frac{\sqrt{3}}{2}(\alpha-1)^{-1}\left\|\left(Q_{0}-z\right)^{-1}\right\| .
$$

Since the eigenvalues of $Q_{0}$ are $\left\{\left(\frac{1}{2}+n\right) \sqrt{\alpha^{2}-1}\right\}_{n=0}^{\infty}$ we have $\sup _{z \in \partial C}\left\|Q_{0}\left(Q_{0}-z\right)^{-1}\right\|$ $=3$ and $\sup _{z \in \partial C}\left\|\left(Q_{0}-z\right)^{-1}\right\|=2 / \sqrt{\alpha^{2}-1}$. Then the lemma follows.

The two-dimensional subspace spanned by the eigenvectors associated with the eigenvalues $\lambda_{1}$ and $\lambda_{2}$ is denoted by $\mathscr{F}$. The orthogonal projection onto $\mathscr{F}$ is then given by

$$
P=P(\varepsilon)=-\frac{1}{2 \pi i} \oint_{\partial C}(Q-z)^{-1} d z
$$


We expand $P(\varepsilon)$ with respect to $\varepsilon$ up to the second order:

$$
P=P_{0}+\varepsilon P_{1}+\varepsilon^{2} R,
$$

where $P_{0}$ is the orthogonal projection onto the ground states of $Q_{0}$ and

$$
\begin{gathered}
P_{1}=-\frac{1}{2 \pi i} \oint_{\partial C}\left(Q_{0}-z\right)^{-1} V\left(Q_{0}-z\right)^{-1} d z \\
R=R(\varepsilon)=-\frac{1}{2 \pi i} \oint_{\partial C}\left(Q_{0}-z\right)^{-1} V\left(Q_{0}-z\right)^{-1} V(Q-z)^{-1} d z .
\end{gathered}
$$

LEMMA 2.3. We have $\|R\| \leq g_{2}$ and $\left\|V P_{1}\right\| \leq E_{0} g_{1}^{2}$.

Proof. By Lemma 2.2, we have

$$
\|R\| \leq \frac{|C|}{2 \pi} \sup _{z \in \partial C}\left\|V\left(Q_{0}-z\right)^{-1}\right\|^{2}\left\|(Q-z)^{-1}\right\| \leq \frac{\sqrt{\alpha^{2}-1}}{2} g_{1}^{2}\left\|\left(Q-\sqrt{\alpha^{2}-1}\right)^{-1}\right\| .
$$

Since $\lambda_{3} \geq \frac{3}{2} \sqrt{\alpha^{2}-1}$, we have $\left\|\left(Q-\sqrt{\alpha^{2}-1}\right)^{-1}\right\|=\left|\lambda_{2}-\sqrt{\alpha^{2}-1}\right|^{-1}$. Hence, $\|R\| \leq g_{2}$ holds. Similarly one can prove the second bound.

Let $v_{0} \in L^{2}(\mathbb{R})$ be the normalized ground state of $h=\frac{1}{2} p^{2}+\left(\left(\alpha^{2}-1\right) / 2\right) x^{2}$. Namely

$$
v_{0}(x)=\left(\frac{\sqrt{\alpha^{2}-1}}{\pi}\right)^{1 / 4} e^{-\sqrt{\alpha^{2}-1} x^{2} / 2} .
$$

Let $S_{a}$ be the dilation defined by $S_{a} f(x)=(1 / \sqrt{a}) f(a x)$ for $a>0$. We define the unitary operator $U$ on $\mathcal{H}$ by

$$
U=\frac{1}{\sqrt{2}} S_{\sqrt{\alpha}}\left(\begin{array}{cc}
e^{i x^{2} /(2 \alpha)} & 0 \\
0 & e^{-i x^{2} /(2 \alpha)}
\end{array}\right)\left(\begin{array}{cc}
1 & -i \\
1 & i
\end{array}\right) .
$$

Then $U Q_{0} U^{*}=\left(\begin{array}{cc}h & 0 \\ 0 & h\end{array}\right)$ and the vectors $u_{1}=U^{*}\left(\begin{array}{c}v_{0} \\ 0\end{array}\right)$ and $u_{2}=U^{*}\left(\begin{array}{c}0 \\ v_{0}\end{array}\right)$ are twofold ground states of $Q(\alpha, \alpha)$. Since $P$ is a projection onto $\mathscr{F}$, the ground state and the first excited state can be expressed as a linear combination of $P u_{1}$ and $P u_{2}$ as long as both $P u_{1}$ and $P u_{2}$ are linearly independent, which is proven in the lemma below.

Lemma 2.4. Assume that $\varepsilon^{2} g_{2}<1$. Then $P u_{1}$ and $P u_{2}$ are non-zero vectors. Moreover, if $\varepsilon^{2} g_{2}<1 / 2$, then $P u_{1}$ and $P u_{2}$ are linearly independent.

Proof. By (2.22) we have

$$
\left\|P u_{1}\right\|^{2}=1+\varepsilon\left\langle u_{1}, P_{1} u_{1}\right\rangle+\varepsilon^{2}\left\langle u_{1}, R u_{1}\right\rangle .
$$

The second term on the right-hand side above is zero, since

$$
\left\langle u_{1}, P_{1} u_{1}\right\rangle=\frac{-1}{2 \pi i} \oint_{\partial C} \frac{1}{\left(E_{0}-z\right)^{2}} d z\left\langle u_{1}, V u_{1}\right\rangle=0 .
$$

Hence, $\left\|P u_{1}\right\|^{2} \geq 1-\varepsilon^{2} g_{2}^{2}>0$ holds by Lemma 2.3 and the assumption. Since the same holds for $\left\|P u_{2}\right\|$, thus $P u_{i}(i=1,2)$ are non-zero vectors. Next we assume that $\varepsilon^{2} g_{2}<1 / 2$. 
Then we have

$$
\begin{aligned}
\left\|P u_{1}\right\|^{2}\left\|P u_{2}\right\|^{2}-\left|\left\langle P u_{1}, P u_{2}\right\rangle\right|^{2} & \geq\left(1-\varepsilon^{2} g_{2}\right)\left(1-\varepsilon^{2} g_{2}\right)-\varepsilon^{4} g_{2}^{2} \\
& =1-2 \varepsilon^{2} g_{2}>0
\end{aligned}
$$

which implies that $P u_{1}$ and $P u_{2}$ are linearly independent.

LEMMA 2.5. Let $g_{3}$ and $g_{4}$ be given in (1.10) and (1.11), respectively. Then it follows that where we used the bound

$$
\begin{aligned}
& \left\|V u_{i}\right\| \leq g_{1} E_{0}, \quad i=1,2, \\
& \left|\left\langle u_{1}, V u_{1}\right\rangle\right|=g_{3}, \\
& \left|\left\langle u_{1}, V u_{2}\right\rangle\right| \leq g_{4} .
\end{aligned}
$$

Proof. Equation (2.31) follows from

$$
\left\|V u_{i}\right\| \leq\left\|V\left(Q_{0}-\sqrt{\alpha^{2}-1}\right)^{-1}\right\|\left\|\left(Q_{0}-\sqrt{\alpha^{2}-1}\right) u_{i}\right\| \leq g_{1} E_{0} .
$$

The proofs of (2.32) and (2.33) are given in the Appendix.

Proof of Theorem 1.4. Suppose that $\varepsilon^{2} g_{2}<1 / 2$. We define

$$
\Phi_{1}=\frac{P u_{1}}{\left\|P u_{1}\right\|}, \quad \Phi_{2}=\frac{P u_{2}-\left\langle\Phi_{1}, u_{2}\right\rangle \Phi_{1}}{\left\|P u_{2}-\left\langle\Phi_{1}, u_{2}\right\rangle \Phi_{1}\right\|} .
$$

Then, by Lemma $2.4, \Phi_{1}$ and $\Phi_{2}$ are orthogonal vectors in $\mathscr{F}$. Let $\mathscr{V}=\operatorname{Span}\left\{\Phi_{1}, \Phi_{2}\right\}$ be the two-dimensional vector space and $Q: \mathscr{V} \rightarrow \mathscr{V}$ can be regarded as a linear operator and its matrix representation is given by

$$
m=\left(\begin{array}{ll}
\left\langle\Phi_{1}, Q \Phi_{1}\right\rangle & \left\langle\Phi_{1}, Q \Phi_{2}\right\rangle \\
\left\langle\Phi_{2}, Q \Phi_{1}\right\rangle & \left\langle\Phi_{2}, Q \Phi_{2}\right\rangle
\end{array}\right)
$$

Thus, the eigenvalues $\lambda_{1}$ and $\lambda_{2}$ of $Q$ are also the eigenvalue of $m$. Therefore, the difference of $\lambda_{1}$ and $\lambda_{2}$ can be computed by

$$
\left(\lambda_{2}-\lambda_{1}\right)^{2}=\left(\left\langle\Phi_{1}, Q \Phi_{1}\right\rangle-\left\langle\Phi_{2}, Q \Phi_{2}\right\rangle\right)^{2}+4\left|\left\langle\Phi_{1}, Q \Phi_{2}\right\rangle\right|^{2},
$$

which implies that

$$
\left|\lambda_{2}-\lambda_{1}\right| \geq 2\left|\left\langle\Phi_{1}, Q \Phi_{2}\right\rangle\right|
$$

We estimate $\left|\left\langle\Phi_{1}, Q \Phi_{2}\right\rangle\right|$ from below. Inserting the definition of $\Phi_{j}$ into $\left\langle\Phi_{i}, Q \Phi_{j}\right\rangle$ we have

$$
\left\langle\Phi_{1}, Q \Phi_{2}\right\rangle=\frac{\left\langle P u_{1}, Q\left(\left\|P u_{1}\right\|^{2} P u_{2}-\left(P u_{1}, P u_{2}\right) P u_{1}\right)\right\rangle}{\left\|P u_{1}\right\|^{3}\left\|P u_{2}-\left(P u_{1}, P u_{2}\right) P u_{1} /\right\| P u_{1}\left\|^{2}\right\|} .
$$

Note that

$$
\left\langle P u_{i}, Q P u_{j}\right\rangle=\left(P u_{i}, Q u_{j}\right)=E_{0} \delta_{i j}+\varepsilon\langle V\rangle_{i j}+\varepsilon^{2}\left(E_{0}\langle R\rangle_{i j}+\left\langle P_{1} V\right\rangle_{i j}\right)+\varepsilon^{3}\langle R V\rangle_{i j},
$$

where $\langle K\rangle_{i j}=\left(u_{i}, K u_{j}\right)$. We see that the denominator of (2.39) is expanded as

$$
\begin{aligned}
& \left\|P u_{1}\right\|\|\| P u_{1}\left\|^{2} P u_{2}-\left(P u_{1}, P u_{2}\right) P u_{1}\right\| \\
& \quad=\left(1+\varepsilon^{2}\langle R\rangle_{11}\right) \sqrt{\left(1+\varepsilon^{2}\langle R\rangle_{11}\right)\left(1+\varepsilon^{2}\langle R\rangle_{22}\right)-\varepsilon^{4}\left|\langle R\rangle_{12}\right|^{2}} .
\end{aligned}
$$


By the bound $\|R\| \leq g_{2}$ we have the lower bound

$$
\left\|P u_{1}\right\|\|\| P u_{1}\left\|^{2} P u_{2}-\left(P u_{1}, P u_{2}\right) P u_{1}\right\| \geq\left(1-\varepsilon^{2} g_{2}\right) \sqrt{1-2 \varepsilon^{2} g_{2}^{2}} .
$$

The numerator of (2.39) can be also expanded as

$$
\begin{aligned}
\left(P u_{1}, Q\left(\left\|P u_{1}\right\|^{2} P u_{2}-\left(P u_{1}, P u_{2}\right) P u_{1}\right)\right) \\
=\varepsilon\langle V\rangle_{12}+\varepsilon^{2}\left\langle P_{1} V\right\rangle_{12}+\varepsilon^{3}\left(\langle R V\rangle_{12}+\langle V\rangle_{12}\langle R\rangle_{11}-\langle R\rangle_{12}\langle V\rangle_{11}\right) \\
\quad+\varepsilon^{4}\left(\left\langle P_{1} V\right\rangle_{12}\langle R\rangle_{11}-\langle R\rangle_{12}\left\langle P_{1} V\right\rangle_{11}\right)+\varepsilon^{5}\left(\langle R\rangle_{11}\langle R V\rangle_{12}-\langle R\rangle_{12}\langle R V\rangle_{12}\right) .
\end{aligned}
$$

By using Lemmas 2.3 and 2.5, each term can be evaluated as

$$
\begin{aligned}
& \varepsilon^{2}\left|\left\langle P_{1} V\right\rangle_{12}\right| \leq \varepsilon^{2} E_{0} g_{1}^{2} \\
& \varepsilon^{3}\left|\langle R V\rangle_{12}+\langle V\rangle_{12}\langle R\rangle_{11}-\langle R\rangle_{12}\langle V\rangle_{11}\right| \leq \varepsilon^{3}\left(E_{0} g_{1} g_{2}+g_{2} g_{4}+g_{2} g_{3}\right) \\
& \varepsilon^{4}\left|\left\langle P_{1} V\right\rangle_{12}\langle R\rangle_{11}-\langle R\rangle_{12}\left\langle P_{1} V\right\rangle_{11}\right| \leq \varepsilon^{4} 2 E_{0} g_{1}^{2} g_{2} \\
& \varepsilon^{5}\left|\langle R\rangle_{11}\langle R V\rangle_{12}-\langle R\rangle_{12}\langle R V\rangle_{12}\right| \leq \varepsilon^{5} 2 E_{0} g_{1} g_{2}^{2} .
\end{aligned}
$$

By combining all of the estimates stated above, we have

$$
\left|\lambda_{1}-\lambda_{2}\right| \geq \frac{2 \varepsilon}{\ell(\varepsilon)}\left(g_{4}-\varepsilon \kappa(\varepsilon)\right),
$$

where $\ell(\varepsilon)=\left(1-\varepsilon^{2} g_{2}\right) \sqrt{1-2 \varepsilon^{2} g_{2}^{2}}$. Hence, the theorem follows.

Acknowledgements. We thank Takashi Ichinose and Masato Wakayama for bringing [IW07] to our attention and giving helpful comments. The work of IS was partially supported by Y22740087 from JSPS, and was performed through the Program for Dissemination of Tenure-Track System funded by the Ministry of Education and Science, Japan. FH is grateful for the financial support by Grant-in-Aid for Science Research (B) 20340032 from JSPS.

\section{A. Appendix: computation of $\langle V\rangle_{i j}$}

We recall that $v_{0}=(\omega / \pi)^{1 / 4} e^{-\omega x^{2} / 2}$ with $\omega=\sqrt{\alpha^{2}-1}, V=\frac{1}{2}\left(\begin{array}{ll}0 & 0 \\ 0 & 1\end{array}\right)\left(p^{2}+x^{2}\right)$ and $U$ is given by (2.27). We directly see that

$$
\begin{aligned}
U V U^{-1}= & \frac{1}{4} S_{\sqrt{\alpha}}\left(\begin{array}{cc}
e^{i x^{2} / 2 \alpha} & 0 \\
0 & e^{-i x^{2} / 2 \alpha}
\end{array}\right)\left(\begin{array}{cc}
1 & -i \\
1 & i
\end{array}\right)\left(\begin{array}{cc}
0 & 0 \\
0 & 1
\end{array}\right)\left(\begin{array}{cc}
1 & 1 \\
i & -i
\end{array}\right) \\
& \times\left(p^{2}+x^{2}\right)\left(\begin{array}{cc}
e^{-i x^{2} / 2 \alpha} & 0 \\
0 & e^{i x^{2} / 2 \alpha}
\end{array}\right) S_{1 / \sqrt{\alpha}} \\
= & \frac{1}{4}\left(\begin{array}{cc}
e^{i x^{2} / 2 \alpha}\left(\frac{p^{2}}{\alpha}+\alpha x^{2}\right) e^{-i x^{2} / 2 \alpha} & e^{-i x^{2} / 2 \alpha}\left(\frac{p^{2}}{\alpha}+\alpha x^{2}\right) e^{i x^{2} / 2 \alpha} \\
-e^{-i x^{2} / 2 \alpha}\left(\frac{p^{2}}{\alpha}+\alpha x^{2}\right) e^{-i x^{2} / 2 \alpha} & e^{i x^{2} / 2 \alpha}\left(\frac{p^{2}}{\alpha}+\alpha x^{2}\right) e^{i x^{2} / 2 \alpha}
\end{array}\right) .
\end{aligned}
$$


Then we have

$$
\begin{aligned}
& \left\langle\left(\begin{array}{l}
1 \\
0
\end{array}\right), U V U^{-1}\left(\begin{array}{l}
1 \\
0
\end{array}\right)\right\rangle_{\mathbb{C}^{2}}=\frac{1}{4} e^{i x^{2} / 2 \alpha}\left(\frac{p^{2}}{\alpha}+\alpha x^{2}\right) e^{-i x^{2} / 2 \alpha} \\
& \left\langle\left(\begin{array}{l}
1 \\
0
\end{array}\right), U V U^{-1}\left(\begin{array}{l}
0 \\
1
\end{array}\right)\right\rangle_{\mathbb{C}^{2}}=-\frac{1}{4} e^{i x^{2} / 2 \alpha}\left(\frac{p^{2}}{\alpha}+\alpha x^{2}\right) e^{i x^{2} / 2 \alpha} .
\end{aligned}
$$

LemmA A.1. We have that $\left\langle u_{1}, V u_{1}\right\rangle=\alpha / 4 \omega$, and then $\left|\left\langle u_{1}, V u_{1}\right\rangle\right|=g_{3}$.

Proof. By (A.1) we have

$$
\left\langle u_{1}, V u_{1}\right\rangle=\frac{1}{4}\left\langle v_{0}, e^{i x^{2} / 2}\left(\frac{p^{2}}{\alpha}+\alpha x^{2}\right) e^{-i x^{2} / 2} v_{0}\right\rangle=\frac{1}{4}\left\langle v_{0},\left(\frac{(p-x)^{2}}{\alpha}+\alpha x^{2}\right) v_{0}\right\rangle .
$$

Since $\left\langle v_{0},(p x+x p) v_{0}\right\rangle=0$, we obtain that

$$
\left\langle u_{1}, V u_{1}\right\rangle=\frac{1}{4}\left\langle v_{0},\left(\frac{p^{2}+x^{2}}{\alpha}+\alpha x^{2}\right) v_{0}\right\rangle .
$$

By $\left\langle v_{0}, x^{2} v_{0}\right\rangle=1 /(2 \omega)$ and $\left\langle v_{0}, p^{2} v_{0}\right\rangle=\omega / 2$ we have $\left\langle u_{1}, V u_{1}\right\rangle=\alpha /(4 \omega)$.

LEMMA A.2. We have that $\left\langle u_{1}, V u_{2}\right\rangle=-\left(\omega^{3 / 2} / 4 \alpha\right)(\omega-i)^{-1 / 2}$. In particular $\left|\left\langle u_{1}, V u_{2}\right\rangle\right|$ $\leq \omega^{3 / 2} / 4 \alpha^{3 / 2}$.

Proof. By (A.2) we have

$$
\begin{aligned}
\left\langle u_{1}, V u_{2}\right\rangle & =-\frac{1}{4}\left\langle v_{0}, e^{i x^{2} / 2}\left(\frac{p^{2}}{\alpha}+\alpha x^{2}\right) e^{i x^{2} / 2} v_{0}\right\rangle \\
& =-\frac{1}{4 \alpha}\left\langle(-i-\omega) x e^{-i x^{2} / 2} v_{0},(i-\omega) x e^{i x^{2} / 2} v_{0}\right\rangle-\frac{\alpha}{4}\left\langle v_{0}, x^{2} e^{i x^{2}} v_{0}\right\rangle \\
& =-\frac{1}{4 \alpha}\left((\omega-i)^{2}+\alpha^{2}\right)\left\langle v_{0}, x^{2} e^{i x^{2}} v_{0}\right\rangle .
\end{aligned}
$$

Since $\left\langle v_{0}, x^{2} e^{i x^{2}} v_{0}\right\rangle=(\omega / \pi)^{1 / 2} \int_{\mathbb{R}} x^{2} e^{-(\omega-i) x^{2}} d x=\frac{1}{2} \omega^{1 / 2}(\omega-i)^{-3 / 2}$, we have the lemma.

\section{REFERENCES}

[GJ68] J. Glimm and A. Jaffe. The $\lambda\left(\phi^{4}\right)_{2}$ quantum field theory without cutoffs. I. Phys. Rev. 176 (1968), 1945-1951.

[HH12] M. Hirokawa and F. Hiroshima. Absence of energy level crossing for the ground state energy of the Rabi model. Preprint, 2012.

[Hir05] F. Hiroshima. Multiplicity of ground states in quantum field models: applications of asymptotic fields. J. Funct. Anal. 224 (2005), 431-470.

[IW05a] T. Ichinose and M. Wakayama. Zeta functions for the spectrum of the non-commutative harmonic oscillators. Commun. Math. Phys. 258 (2005), 697-739.

[IW05b] T. Ichinose and M. Wakayama. Special values of the spectral zeta function of the non-commutative harmonic oscillator and confluent Heun equations. Kyushu J. Math. 59 (2005), 39-100.

[IW07] T. Ichinose and M. Wakayama. On the spectral zeta function for the non-commutative harmonic oscillator. Rep. Math. Phys. 59 (2007), 421-432.

[KW06] K. Kimoto and M. Wakayama. Apéry-like number arising from special values of spectral zeta functions for non-commutative harmonic oscillators. Kyushu J. Math. 60 (2006), 383-404. 
[KW07] K. Kimoto and M. Wakayama. Elliptic curves arising from the spectral zeta function for noncommutative harmonic oscillators and $\Gamma_{0}(4)$-modular forms. The Conference on L-Functions. World Scientific, Hackensack, NJ, 2007, pp. 201-218.

[KY09] K. Kimoto and Y. Yamasaki. A variation of multiple $L$-values arising from the spectral zeta function of the non-commutative harmonic oscillator. Proc. Amer. Math. Soc. 137 (2009), 2503-2515.

[NNW02] K. Nagatou, M. T. Nakao and M. Wakayama. Verified numerical computations for eigenvalues of non-commutative harmonic oscillators. Num. Funct. Anal. and Opt. 23 (2002), 633-650.

[Och01] H. Ochiai. Non-commutative harmonic oscillators and Fuchsian ordinary differential operators. Commun. Math. Phys. 217 (2001), 357-373.

[Och04] H. Ochiai. Non-commutative harmonic oscillators and the connection problem for the Heun differential equation. Lett. Math. Phys. 70 (2004), 133-139.

[PW01] A. Parmeggiani and M. Wakayama. Oscillator representations and systems of ordinary differential equations. Proc. Natl. Acad. Sci. USA 98 (2001), 26-30.

[PW02a] A. Parmeggiani and M. Wakayama. Non-commutative harmonic oscillator I. Formum Math. 14 (2002), 539-604.

[PW02b] A. Parmeggiani and M. Wakayama. Non-commutative harmonic oscillator II. Formum Math. 14 (2002), 669-690.

[PW03] A. Parmeggiani and M. Wakayama. Corrigenda and remarks to: "Non-commutative harmonic oscillators. I.” [Forum Math. 14 (2002), 539-604]. Forum Math. 15 (2003), 955-963.

[Par04] A. Parmeggiani. On the spectrum and the lowest eigenvalue of certain non-commutative harmonic oscillators. Kyushu J. Math. 58 (2004), 277-322.

[Par06] A. Parmeggiani. On the spectrum of certain noncommutative harmonic oscillators. Ann. Univ. Ferrara Sez. VII Sci. Mat. 52 (2006), 431-456.

[Par08a] A. Parmeggiani. On the spectrum of certain non-commutative harmonic oscillators and semiclassical analysis. Comm. Math. Phys. 279 (2008), 285-308.

[Par08b] A. Parmeggiani. Introduction to the spectral theory of non-commutative harmonic oscillators (COE Lecture Note, 8). Kyushu University, Kyushu, 2008.

[Par10] A. Parmeggiani. Spectral theory of non-commutative harmonic oscillators: an introduction (Lecture Notes in Mathematics, 1992), Springer, Berlin, 2010.

\section{Fumio Hiroshima \\ Faculty of Mathematics \\ Kyushu University \\ Fukuoka 819-0395 \\ Japan}

(E-mail: hiroshima@math.kyushu-u.ac.jp)

Itaru Sasaki

Fiber-Nanotech Young Researcher Empowerment Center

Shinshu University

Matsumoto 390-8621

Japan

(E-mail: isasaki@shinshu-u.ac.jp) 